\title{
Functional prostate-specific membrane antigen is enriched in exosomes from prostate cancer cells
}

\author{
TIANCHENG LIU, DESIREE E. MENDES and CLIFFORD E. BERKMAN \\ Department of Chemistry, Washington State University, Pullman, WA 99164, USA
}

Received September 21, 2013; Accepted November 4, 2013

DOI: 10.3892/ijo.2014.2256

\begin{abstract}
Developing simple and effective approaches to detect tumor markers will be critical for early diagnosis or prognostic evaluation of prostate cancer treatment. Prostate-specific membrane antigen (PSMA) has been validated as an important tumor marker for prostate cancer progression including angiogenesis and metastasis. As a type II membrane protein, PSMA can be constitutively internalized from the cell surface into endosomes. Early endosomes can fuse with multivesicular bodies (MVB) to form and secrete exosomes (40-100 nm) into the extracellular environment. Herein, we tested whether some of the endosomal PSMA could be transferred to exosomes as an extracellular resource for PSMA. Using PSMA-positive LNCaP cells, the secreted exosomes were collected and isolated from the cultured media. The vesicular structures of exosomes were identified by electron microscopy, and exosomal marker protein CD9 and tumor susceptibility gene (TSG 101) were confirmed by western blot analysis. Our present data demonstrate that PSMA can be enriched in exosomes, exhibiting a higher content of glycosylation and partial proteolysis in comparison to cellular PSMA. An in vitro enzyme assay further confirmed that exosomal PSMA retains functional enzymatic activity. Therefore, our data may suggest a new role for PSMA in prostate cancer progression, and provide opportunities for developing non-invasive approaches for diagnosis or prognosis of prostate cancer.
\end{abstract}

\section{Introduction}

Prostate cancer remains the second leading cause of cancer death for men in the US. According to the National Cancer Institute, it is estimated that there will be 238,590 new cases and 29,720 deaths from prostate cancer in 2013 (http://www. cancer.gov/cancertopics/types/prostate). The cell-surface

Correspondence to: Professor Clifford E. Berkman, Department of Chemistry, Washington State University, Pullman, WA 99164-4630, USA

E-mail: cberkman@wsu.edu

Key words: prostate-specific membrane antigen, exosome, biomarker, glycosylation, prostate cancer enzyme prostate-specific membrane antigen (PSMA) is upregulated and strongly expressed on prostate cancer cells, including those that are metastatic (1). Endothelial-expression of prostate-specific membrane antigen (PSMA) in the neovasculature of a variety of non-prostatic solid malignancies has also been reported $(2,3)$. PSMA is a type II membrane protein, consisting of a cytoplasmic domain (1-19aa), transmembrane domain (20-44aa) and extracellular domain (45-750aa), exhibits both $\mathrm{N}$-acetylated $\alpha$-linked acidic dipeptidase (NAALADase) and folate hydrolase (FOLH) activities, and constitutive or induced internalization (4-6). These properties have allowed PSMA to attract considerable attention as a target for antibody or small molecule inhibitor-guided delivery of imaging and therapeutic agents toward prostate cancer (7-15). Furthermore, pilot studies support the position that PSMA is an ideal biomarker for the targeted imaging and therapy of PSMA-positive prostate cancer.

Exosomes are small vesicles $(40-100 \mathrm{~nm})$ secreted by multiple normal tissue or pathological cells including cancers containing proteins, mRNAs, microRNAs and lipids that are from original cells (16). Through exosome-carrying messages, cells can achieve cross-talk without contacting each other (17). It was noted that there are elevated exosome levels secreted by highly advanced cancer cells enriched with tumor-markers (18). These studies may suggest that tumor-secreting exosomes may play an important role in the development and progression of cancer, serving as a potential biomarker resource to develop non-invasive and dynamic approaches for tumor diagnosis and prognostic evaluation of cancer treatment (16-19).

In the present study, we sought to determine the extent of PSMA enrichment in exosomes secreted by human prostate cancer cells (PSMA-positive LNCaP), and whether the exosomal PSMA is functionally active. Herein, our data revealed that tumor-marker PSMA is strongly enriched in exosomes secreted by PSMA-positive prostate cancer cells, and the exosomal PSMA maintains its functional enzymatic activity despite of higher glycosylation content. Therefore, tumor-related exosomal PSMA may serve as a diagnostic or prognostic biomarker for prostate cancer.

\section{Materials and methods}

Cell lines and reagents. The human prostate cancer cell line LNCaP was obtained from the American Type Culture Collection (Manassas, VA, USA). CWR22Rv1 cells were 
obtained from Professor Henry F. VanBrocklin (University of California, San Francisco, CA, USA). Mouse monoclonal anti-human EpCAM antibody was obtained from Cell Signaling Technology (Danvers, MA, USA). Mouse monoclonal anti-TSG 101 antibody (C-2) was obtained from Santa Cruz Biotechnology (Santa Cruz, CA, USA). Mouse monoclonal anti-GAPDH antibody and anti- $\alpha$-tubulin antibody were obtained from Sigma-Aldrich (St. Louis, MO, USA). Mouse monoclonal anti-CD9 antibody (LT-86A) was a gift of Dr Davis at Washington State University (Pullman, WA, USA). Mouse monoclonal anti-PSMA antibody 7E11 was graciously provided by Cytogen Corporation (Princeton, NJ, USA). PNGase F was obtained from New England Biolabs (Ipswich, MA, USA). Halt Protease Inhibitor Cocktail (100X) was purchased from Thermo Fisher Scientific (Rockford, IL, USA). All other chemicals and cell-culture reagents were purchased from Fisher Scientific (Sommerville, NJ, USA) or Sigma-Aldrich.

Cell culture. LNCaP and CWR22Rv1 were grown in T-75 flasks with normal growth media [RPMI-1640 containing $10 \%$ heat-inactivated fetal bovine serum (FBS), 100 units of penicillin and $100 \mu \mathrm{g} / \mathrm{ml}$ streptomycin] in a humidified incubator at $37^{\circ} \mathrm{C}$ with $5 \% \mathrm{CO}_{2}$. Confluent cells were detached with a $0.25 \%$ trypsin $0.53 \mathrm{mM}$ EDTA solution for subculture growth.

Exosome isolation. Twenty flasks (each cell line) of prostate cancer cells at $80 \%$ confluence (3 days), were subjected to washing twice in $5 \mathrm{ml}$ serum-free RPMI-1640 media, then replaced with $10 \mathrm{ml}$ serum-free RPMI-1640 media to continue growth for $48 \mathrm{~h}$. A total of $100 \mathrm{ml}$ of cell conditioned media were centrifuged at $300 \mathrm{x} \mathrm{g}$ for $10 \mathrm{~min}$ at $4^{\circ} \mathrm{C}$ to pellet the suspension cells. The supernatant media was further cleared by centrifugation at $16,500 \times \mathrm{g}$ at $4^{\circ} \mathrm{C}$ for $30 \mathrm{~min}$ to remove protein aggregates and cell debris. The collected supernatant was passed through $0.22 \mu \mathrm{m}$ filter to remove the $>200 \mathrm{~nm}$ protein aggregates or vesicles. The filtered media was concentrated to $60 \mathrm{ml}$ using a $70 \mathrm{kDa}$ MWCO Centricon Plus-20 filter capsule (Millipore, Billerica, MA, USA). The concentrated media were transferred to an ultracentrifuge tube for centrifugation at $100,000 \mathrm{x} \mathrm{g}$ for $70 \mathrm{~min}$ at $4^{\circ} \mathrm{C}$ to pellet exosomes. The isolated exosomes were rinsed in $10 \mathrm{ml}$ of PBS buffer, and centrifuged at $100,000 \times \mathrm{g}$ for $1 \mathrm{~h}$ at $4^{\circ} \mathrm{C}$ to be applied for the following experiments.

Transmission electron microscopy (TEM). The isolated exosomes from LNCaP cells were fixed with $50 \mu 1$ of $4 \%$ formaldehyde for $15 \mathrm{~min}$ at room temperature, and $5 \mu \mathrm{l}$ of sample was loaded onto carbon-coated copper grids and left for $20 \mathrm{~min}$ at room temperature. The sample was washed three times in PBS and then fixed for 5 min in $1 \%$ glutaraldehyde. After three washes, the exosome sample was stained for 10 min with saturated aqueous uranyl, and dried after removal of excess liquid. The samples were observed in a FEI Tecnai T-20 at $200 \mathrm{kV}$ and images were recorded using iTEM software (Olympus, Münster, Germany).

Exosomal protein extraction and western blot analysis. For protein extraction, the isolated exosomes were re-suspended in $50 \mu \mathrm{l}$ of lysis buffer (1\% NP-40, $20 \mathrm{mM}$ Tris $\mathrm{pH} 8.0$,
$137 \mathrm{mM} \mathrm{NaCl}, 10 \%$ glycerol) supplemented with 1X Halt Protease Inhibitor Cocktail, kept on ice for $15 \mathrm{~min}$, then centrifuged at $10,000 \mathrm{x} \mathrm{g}$ for $15 \mathrm{~min}$. The supernatant was collected, and stored at $-80^{\circ} \mathrm{C}$. The whole-cell protein extraction was also performed as controls, according to our previous protocol (20-22). Protein concentrations were determined using Non-Interfering Protein Assay (G-Biosciences, St. Louis, MO, USA). Western blot analysis was performed as described previously with only minor modifications $(22,23)$. In brief, cellular proteins $(30 \mu \mathrm{g})$ and exosomal proteins $(5 \mu \mathrm{g})$ were loaded and separated on a NuPAGE ${ }^{\mathrm{TM}} 4-12 \%$ Bis-Tris Gel (Invitrogen, Carlsbad, CA, USA) by electrophoresis for $60 \mathrm{~min}$ at a constant $200 \mathrm{~V}$ under reducing conditions, and then transferred to a $0.45-\mu \mathrm{m}$ PVDF Immobilon-P Transfer Membrane (Millipore Corporation, Bedford, MA, USA) at $400 \mathrm{~mA}$ for $120 \mathrm{~min}$ in a transfer apparatus-Owl Bandit VEP-2 (Owl, Portsmouth, NH, USA) according to the manufacturer's instructions. Membranes were incubated with primary antibody at corresponding dilution overnight at $4^{\circ} \mathrm{C}$ and then with horseradish peroxidase conjugated-second antibody for $1 \mathrm{~h}$ at room temperature. The immunoreactive bands were visualized using Protein Detector TMB Western Blot Kit (KPL, Gaithersburg, MD, USA) following the manufacturer's instructions.

Deglycosylation analysis of PSMA. According to manufacturer's guidance, cellular PSMA and exosomal PSMA were subjected to denaturation in $1 \mathrm{X}$ denaturing buffer for $10 \mathrm{~min}$ at $100^{\circ} \mathrm{C}$, cooled and spun down. The denatured proteins were mixed with $\mathrm{PNGase} \mathrm{F}$ in $1 \mathrm{X}$ reaction buffer containing $1 \% \mathrm{NP}-40$ to incubate for $3 \mathrm{~h}$ at $37^{\circ} \mathrm{C}$. Suitable amounts of deglycosylated PSMAs were analyzed by western blotting, the equal amounts of intact cellular PSMA and exosomal PSMA were loaded as controls.

Enzymatic activity analysis. HPLC-based PSMA enzymatic activity analysis was performed in triplicate as described previously with only minor modifications $(6,24)$. Working solutions of the substrate $\{\mathrm{N}$-[4-(phenylazo)-benzoyl]glutamyl- $\gamma$-glutamic acid, (PABG $\gamma \mathrm{G})\}$ were made at $10 \mu \mathrm{M}$ in Tris buffer (50 mM, pH 7.4). Working solutions of each protein sample were diluted at suitable concentrations in Tris buffer (50 mM, pH 7.4 containing 1\% Triton X-100) to obtain $\sim 15 \%$ conversion (product/total substrate). A typical incubation mixture (final volume $250 \mu \mathrm{l}$ ) was prepared by the addition of $175 \mu 1$ Tris buffer (50 mM, pH 7.4) and PABG $\gamma \mathrm{G}$ $(25 \mu 1,10 \mu \mathrm{M})$ in a test tube. The enzymatic reaction was initiated by the addition of $25 \mu \mathrm{l}$ of the PSMA working solution. The reaction was allowed to proceed for $15 \mathrm{~min}$ with constant shaking at $37^{\circ} \mathrm{C}$ and terminated by the addition of $25 \mu 1$ methanolic TFA (2.5\% trifluoroacetic acid by volume in methanol) followed by vortexing. The quenched incubation mixture was quickly buffered by the addition of $25 \mu \mathrm{K}_{2} \mathrm{HPO}_{4}$ $(0.1 \mathrm{M})$, vortexed, iced for $15 \mathrm{~min}$, and centrifuged (10 min at $7,000 \times \mathrm{g})$. An $85 \mu \mathrm{l}$ aliquot of the resulting supernatant was subsequently quantified for the proportions of substrate and product by HPLC as previously described $(25,26)$. Fractional enzymatic activity for each protein sample was calculated from HPLC data. The relative enzymatic activity (exosomal PSMA/cellular PSMA) was further calculated. 


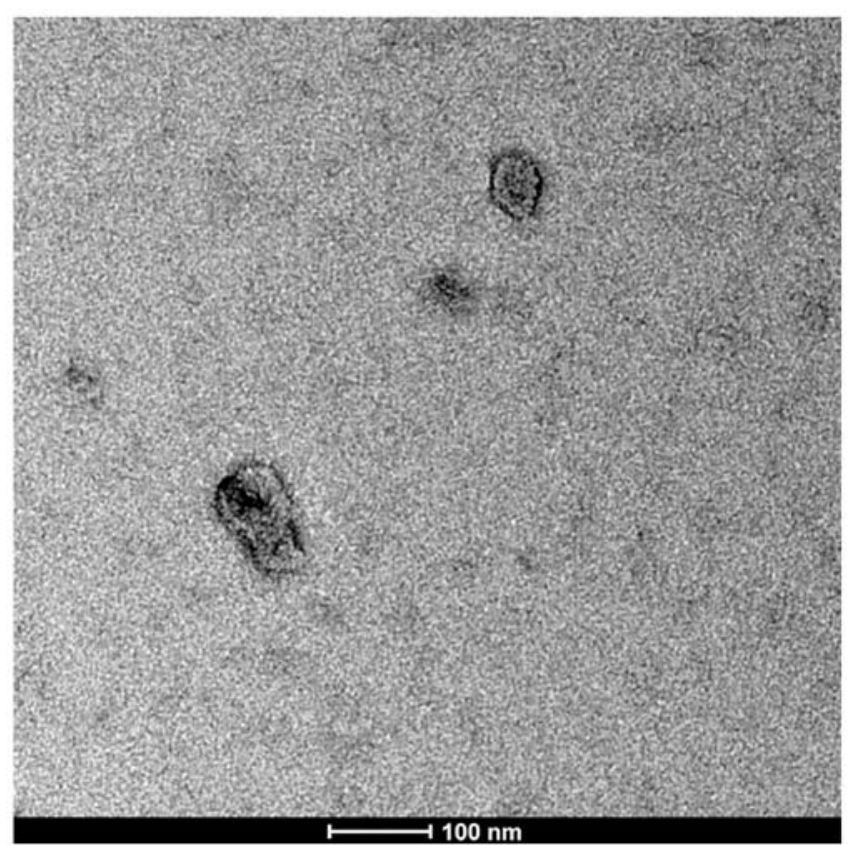

Figure 1. TEM analysis of exosomes from LNCaP cells. Exosomes exhibit classic morphology with round or cup-shaped membrane vesicles (40-100 nm). Distance scale, $100 \mathrm{~nm}$.

\section{Results}

Validation of exosome isolation protocol. TEM analysis clearly revealed that vesicle morphology was cup or round shaped and the size $(<100 \mathrm{~nm})$ was characteristic $(27,28)$ for exosomes (Fig. 1). This result confirmed the efficacy of our protocol for isolating exosomes from LNCaP cells.

Enrichment of PSMA in exosomes. Western blot analysis data (Fig. 2) demonstrated exceptional enrichment of exosomal markers (TSG 101, CD9) and epithelial cell adhesion molecule (EpCAM), as well as moderately enriched prostate tumor-marker PSMA in exosomes when compared to relatively stable $\alpha$-tubulin levels in cells and exosomes. Interestingly, exosomal PSMA was also found to be highly enriched with an increase in molecular weight when compared to the cell extract, and also contained a small amount of proteolytic fragments (Fig. 2).

Highly glycosylated exosomal PSMA. To identify the source of PSMA's perturbed molecular weight, glycosylation analysis of cellular and exosomal PSMAs were performed with PNGase F to remove all N-linked glycosylation from PSMA. After deglycosylation, cellular and exosomal PSMAs exhibited the same size of molecular weight on western blot analysis (Fig. 3).

Retaining enzymatic activity of exosomal PSMA. Equal amounts of exosomal and cellular PSMAs were evaluated for their enzymatic activities using an HPLC-based, in vitro enzyme assay. Exosomal PSMA retains $\sim 24 \%$ enzyme activity of cellular PSMA (Fig. 4), attributed to partial proteolysis (Fig. 2) and lower $\mathrm{pH}$ within endosomes causing denaturation of internalized PSMA during exosome formation.

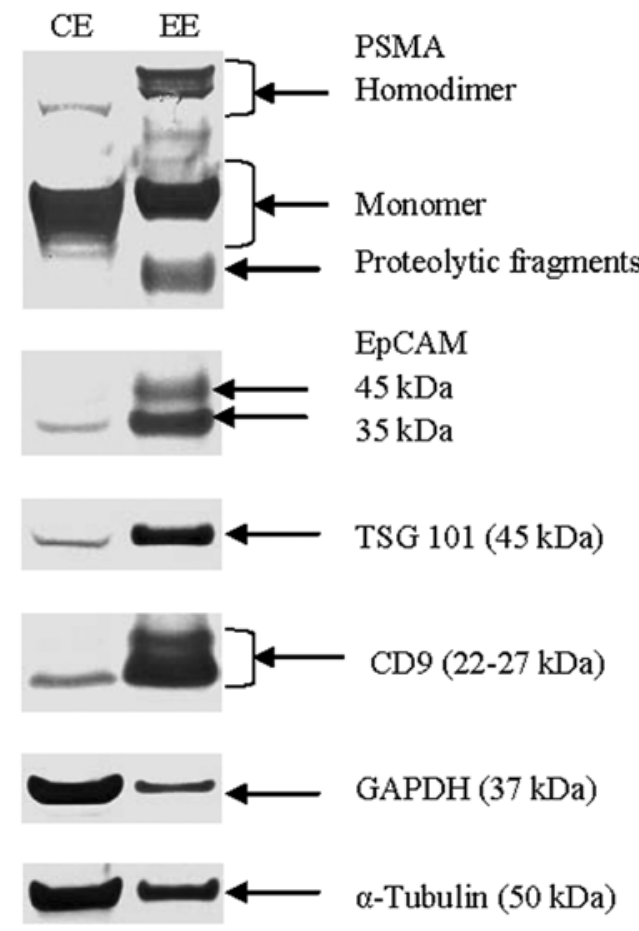

Figure 2. Differently enriched proteins by LNCaP cells and exosomes. The cell extract (CE) and exosome extract (EE) were analyzed by western blotting. The data clearly demonstrated that exosomes were moderately enriched with PSMA, and high levels for exosomal markers (TSG 101, CD9) and EpCAM, a low level of GAPDH and relatively stable for $\alpha$-tubulin, which was detected to serve as a protein loading control.

Confirmation of enriched PSMA in CWR22Rv1-derived exosomes. Employment of another PSMA-positive prostate cancer cell line (CWR22Rv1) through western blot analysis (Fig. 5) further validated that CWR22Rv1-derived exosomes were also enriched with highly glycosylated PSMA analogous to LNCaP-derived exosomes. As controls, the exosomal markers (CD9 and TSG 101) were also highly enriched. Surprisingly, EpCAM was found to be at a low level in CWR22Rv1-derived exosomes, but detected at a higher level in CWR22Rv1 cells.

\section{Discussion}

A plethora of empirical data support that tumor-derived exosomes can serve as cellular representatives or messengers carrying multiple forms of tumor-associated information including signaling molecules, tumor-markers and genetic factors, which may be an untapped potential source of cancer biomarkers for diagnostic or prognostic applications toward multiple cancer types (16). For prostate cancer, our study was carried out to explore whether prostate tumor-derived exosomes were enriched with PSMA, because PSMA has been widely studied and validated as an important biomarker for prostate cancer. Thus, two PSMA-positive prostate cancer cell lines: LNCaP (androgen-dependent) and CWR22Rv1 (androgen-independent) cells were employed in the present project. Although it has been reported that prostate tumor-derived exosomes can enrich biomarker PSMA $(28,29)$, by using both of LNCaP and CWR22Rv1 cells, our data further confirmed the enrichment of exosomal PSMA without regard 


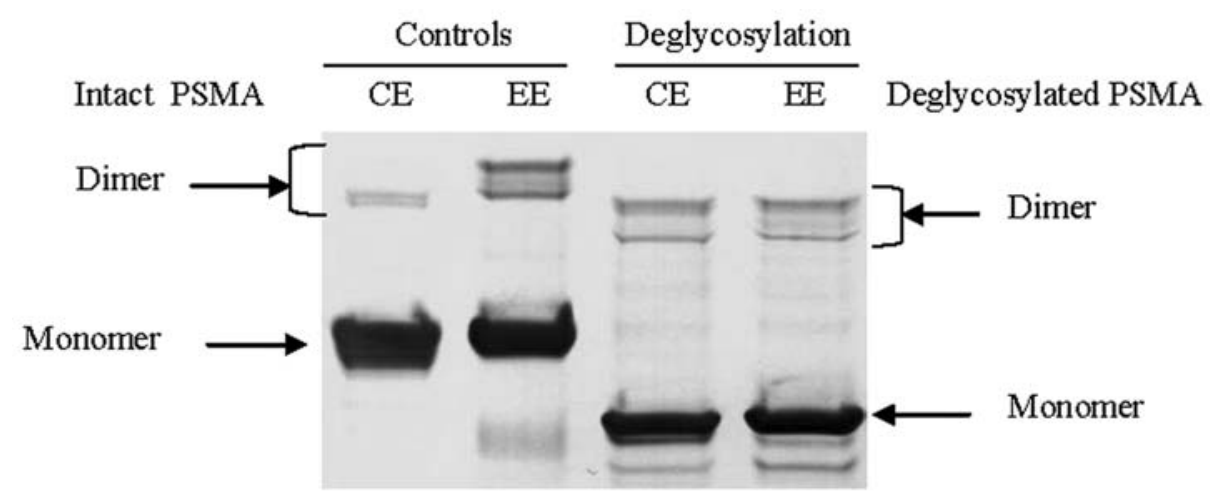

Figure 3. Deglycosylation analysis of cellular and exosomal PSMAs. The cell extract (CE) and exosome extract (EE) proteins were deglycosylated with PNGase F and analyzed by western blotting. Deglycosylated PSMAs exhibit the same size of molecular weight, implicating that high-content glycosylation contributes to the increased molecular weight of exosomal PSMA, compared to cellular PSMA.

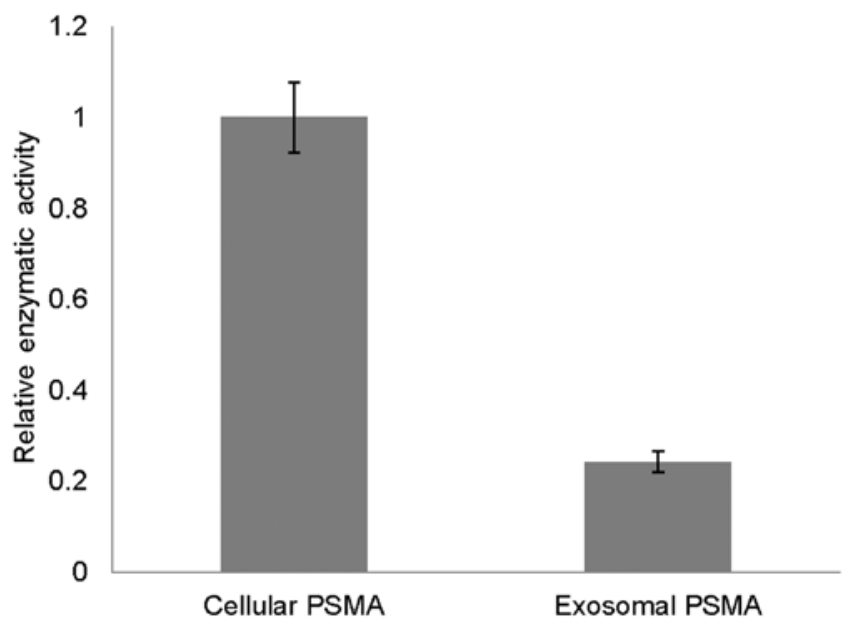

Figure 4. Decreased enzyme activity of exosomal PSMA. Relative enzymatic activity (exosomal PSMA: cellular PSMA at equal protein level) is about 0.24 . The decreased enzyme activity is due to partial proteolysis or low pH-mediated denaturation of internalized PSMA during exosome formation.

to androgen-dependence or -independence of PSMA-positive prostate cancer cells. To our surprise, our data revealed that exosomal PSMA is highly glycosylated, and still retains about $24 \%$ enzymatic activity when compared to cellular PSMA. This evidence suggests that the origin of exosomal PSMA may be from internalization of mature (highly glycosylated) PSMA on the cell surface. The observed diminished activity of PSMA may be due to partial proteolysis or loss of native conformation under the low $\mathrm{pH}$ environment of endosomes; a result of the internalization process prior to fusing with multivesicular bodies (MVB) for exosome formation. Our data also suggest that there may be alternative fates for internalized PSMA: extracellular secretion through exosomes, recycling to the membrane surface or lysosomal digestion $(4,6,30)$.

Currently, there are three major approaches for exosome isolation including ultracentrifugation, chemical precipitation and affinity-binding beads $(31,32)$ which all have shortcomings. The first two approaches are void of specificity, and the last one is dependent on the binding-target protein. In example, EpCAM-based exosome-capture

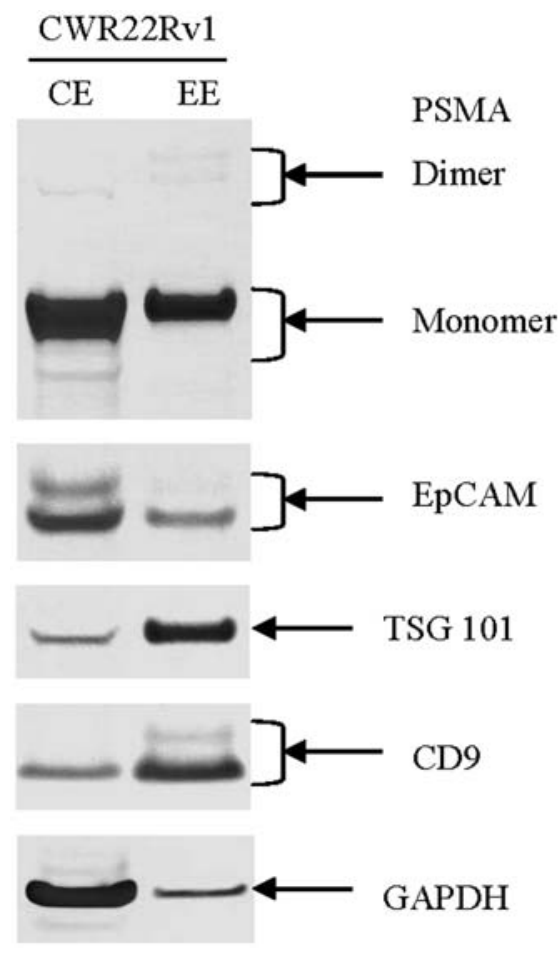

Figure 5. Confirmation of enriched PSMA in CWR22Rv1-derived exosomes. The cell extract (CE) and exosome extract (EE) were analyzed by western blotting. The data clearly demonstrated that CWR22Rv1-derived exosomes were moderately enriched with PSMA in the same manner as LNCaP-derived exosomes, high levels for exosomal markers (TSG 101, CD9), and low levels for EpCAM and GAPDH.

technology is not selective, suffering from contamination of normal tissue-derived exosomes, because EpCAM is widely expressed among a variety of human epithelial tissues, cancers, progenitor and stem cells (33). In contrast, highly-expressed PSMA is only found in prostate cancer cells (34). In fact, our group recently reported successful capture of PSMA-positive prostate cancer cells from blood samples using PSMA-based capture technology (35). Therefore, our data strongly support the development of a novel PSMA-based exosome capture technology platform for the accurate isolation of prostate tumor-derived exosomes from normal tissue-related exosomes. 
In summary, our present data support the concept that prostate tumor-derived exosomes are highly enriched with tumor-marker biomolecules (especially membrane proteins, such as PSMA) representing characteristics of the original prostate cancer cells. Furthermore, characterization of tumor-derived exosomes may provide opportunities for the discovery of novel tumor-related biomarkers. We expect that developing a highly efficient, PSMA-based approach for tumor-derived exosome isolation will accelerate the innovation of non-invasive diagnostic or prognostic technologies for prostate cancer.

\section{Acknowledgements}

The authors thank Cytogen Corporation (Princeton, NJ, USA) and Dr William C. Davis (Veterinary Microbiology and Pathology at WSU, USA) for the gifts of the mouse monoclonal anti-PSMA antibody 7E11 and anti-CD9 antibody, respectively. The authors also extend their gratitude for technical assistance from C. Davitt and V. Lynch-Holm at the WSU Franceschi Microscopy and Imaging Center. This study was supported in part by the National Institutes of Health (R01CA140617).

\section{References}

1. Bacich DJ, Pinto JT, Tong WP and Heston WD: Cloning, expression, genomic localization, and enzymatic activities of the mouse homolog of prostate-specific membrane antigen/ NAALADase/folate hydrolase. Mamm Genome 12: 117-123, 2001.

2. Chang SS, Reuter VE, Heston WD and Gaudin PB: Comparison of anti-prostate-specific membrane antigen antibodies and other immunomarkers in metastatic prostate carcinoma. Urology 57 1179-1183, 2001.

3. Chang SS, O'Keefe DS, Bacich DJ, Reuter VE, Heston WD and Gaudin PB: Prostate-specific membrane antigen is produced in tumor-associated neovasculature. Clin Cancer Res 5: 2674-2681, 1999.

4. Liu H, Rajasekaran AK, Moy P, et al: Constitutive and antibody-induced internalization of prostate-specific membrane antigen. Cancer Res 58: 4055-4060, 1998.

5. Rajasekaran AK, Anilkumar G and Christiansen JJ: Is prostate-specific membrane antigen a multifunctional protein? Am J Physiol Cell Physiol 288: C975-C981, 2005.

6. Liu T, Wu LY, Kazak M and Berkman CE: Cell-surface labeling and internalization by a fluorescent inhibitor of prostate-specific membrane antigen. Prostate 68: 955-964, 2008.

7. Tagawa ST, Beltran H, Vallabhajosula S, et al: Anti-prostatespecific membrane antigen-based radioimmunotherapy for prostate cancer. Cancer 116: 1075-1083, 2010.

8. Wolf P, Freudenberg N, Buhler P, et al: Three conformational antibodies specific for different PSMA epitopes are promising diagnostic and therapeutic tools for prostate cancer. Prostate 70: 562-569, 2010

9. Murphy GP, Greene TG, Tino WT, Boynton AL and Holmes EH: Isolation and characterization of monoclonal antibodies specific for the extracellular domain of prostate specific membrane antigen. J Urol 160: 2396-2401, 1998.

10. Morris MJ, Pandit-Taskar N, Divgi CR, et al: Phase I evaluation of J591 as a vascular targeting agent in progressive solid tumors. Clin Cancer Res 13: 2707-2713, 2007.

11. Milowsky MI, Nanus DM, Kostakoglu L, et al: Vascular targeted therapy with anti-prostate-specific membrane antigen monoclonal antibody J591 in advanced solid tumors. J Clin Oncol 25: 540-547, 2007.

12. Tsukamoto T, Wozniak KM and Slusher BS: Progress in the discovery and development of glutamate carboxypeptidase II inhibitors. Drug Discov Today 12: 767-776, 2007.

13. Kularatne SA, Zhou Z, Yang J, Post CB and Low PS: Design, synthesis, and preclinical evaluation of prostate-specific membrane antigen targeted $(99 \mathrm{~m}) \mathrm{Tc}$-radioimaging agents. Mol Pharm 6: 790-800, 2009.
14. Lapi SE, Wahnishe H, Pham D, et al: Assessment of an 18 F-labeled phosphoramidate peptidomimetic as a new prostate-specific membrane antigen-targeted imaging agent for prostate cancer. J Nucl Med 50: 2042-2048, 2009.

15. Cho SY, Gage KL, Mease RC, et al: Biodistribution, tumor detection, and radiation dosimetry of $18 \mathrm{~F}-\mathrm{DCFBC}$, a lowmolecular-weight inhibitor of prostate-specific membrane antigen, in patients with metastatic prostate cancer. J Nucl Med 53: 1883-1891, 2012.

16. Azmi AS, Bao B and Sarkar FH: Exosomes in cancer development, metastasis, and drug resistance: a comprehensive review. Cancer Metastasis Rev: May 25, 2013 (Epub ahead of print).

17. Thery C: Exosomes: secreted vesicles and intercellular communications. F1000 Biol Rep 3: 15, 2011.

18. Thompson CA, Purushothaman A, Ramani VC, Vlodavsky I and Sanderson RD: Heparanase regulates secretion, composition, and function of tumor cell-derived exosomes. J Biol Chem 288: 10093-10099, 2013.

19. Kharaziha P, Ceder S, Li Q and Panaretakis T: Tumor cell-derived exosomes: a message in a bottle. Biochim Biophys Acta 1826: 103-111, 2012.

20. Liu T, Mendes DE and Berkman CE: From AR to c-Met: Androgen deprivation leads to a signaling pathway switch in prostate cancer cells. Int J Oncol 43: 1125-1130, 2013.

21. Liu T, Wu LY, Fulton MD, Johnson JM and Berkman CE: Prolonged androgen deprivation leads to downregulation of androgen receptor and prostate-specific membrane antigen in prostate cancer cells. Int J Oncol 41: 2087-2092, 2012.

22. Liu T, Wu LY and Berkman CE: Prostate-specific membrane antigen-targeted photodynamic therapy induces rapid cytoskeletal disruption. Cancer Lett 296: 106-112, 2010.

23. Liu T, Toriyabe Y and Berkman CE: Purification of prostate-specific membrane antigen using conformational epitope-specific antibody-affinity chromatography. Protein Expr Purif 49: 251-255, 2006.

24. Wu LY, Anderson MO, Toriyabe Y, et al: The molecular pruning of a phosphoramidate peptidomimetic inhibitor of prostate-specific membrane antigen. Bioorg Med Chem 15: 7434-7443, 2007.

25. Anderson MO, Wu LY, Santiago NM, et al: Substrate specificity of prostate-specific membrane antigen. Bioorg Med Chem 15: 6678-6686, 2007.

26. Maung J, Mallari JP, Girtsman TA, et al: Probing for a hydrophobic a binding register in prostate-specific membrane antigen with phenylalkylphosphonamidates. Bioorg Med Chem 12: 4969-4979, 2004.

27. Lehmann BD, Paine MS, Brooks AM, et al: Senescenceassociated exosome release from human prostate cancer cells. Cancer Res 68: 7864-7871, 2008.

28. Hosseini-Beheshti E, Pham S, Adomat H, Li N and Tomlinson Guns ES: Exosomes as biomarker enriched microvesicles: characterization of exosomal proteins derived from a panel of prostate cell lines with distinct AR phenotypes. Mol Cell Proteomics 11: 863-885, 2012.

29. Mitchell PJ, Welton J, Staffurth J, et al: Can urinary exosomes act as treatment response markers in prostate cancer? J Transl Med 7: 4, 2009.

30. Rajasekaran SA, Anilkumar G, Oshima E, et al: A novel cytoplasmic tail MXXXL motif mediates the internalization of prostate-specific membrane antigen. Mol Biol Cell 14: 4835-4845, 2003.

31. Vlassov AV, Magdaleno S, Setterquist R and Conrad R: Exosomes: current knowledge of their composition, biological functions, and diagnostic and therapeutic potentials. Biochim Biophys Acta 1820: 940-948, 2012.

32. Tauro BJ, Greening DW, Mathias RA, et al: Comparison of ultracentrifugation, density gradient separation, and immunoaffinity capture methods for isolating human colon cancer cell line LIM1863-derived exosomes. Methods 56: 293-304, 2012.

33. Trzpis M, McLaughlin PM, de Leij LM and Harmsen MC: Epithelial cell adhesion molecule: more than a carcinoma marker and adhesion molecule. Am J Pathol 171: 386-395, 2007.

34. Ghosh A and Heston WD: Tumor target prostate specific membrane antigen (PSMA) and its regulation in prostate cancer. J Cell Biochem 91: 528-539, 2004.

35. Wu LY, Liu T, Hopkins MR, Davis WC and Berkman CE: Chemoaffinity capture of pre-targeted prostate cancer cells with magnetic beads. Prostate 72: 1532-1541, 2012. 